Supporting Information for:

\title{
An Experimental and Modeling Comparison of the Dynamics of Capped and Freestanding Poly(2-chlorostyrene) Films
}

Zijian Song, ${ }^{1}$ Ronald P. White, ${ }^{2}$ Jane E. G. Lipson, ${ }^{* 2}$ Simone Napolitano*,1

${ }^{1}$ Polymer and Soft Matter Dynamics, Faculté des Sciences, Université libre de Bruxelles (ULB), Brussels, 1050 Belgium

${ }^{2}$ Department of Chemistry, Dartmouth College, Hanover, NH 03755, USA

*JEGL Jane.E.G.Lipson@dartmouth.edu *SN snapolit@ulb.ac.be

1. Details on CFV model, calculations for P2ClS films, and P2ClS bulk characterization 


\section{Details on CFV model, calculations for P2CIS films, and P2CIS bulk characterization}

The following are some details on the application of the cooperative free volume (CFV) rate model. ${ }^{1-5} \mathrm{CFV}$ is important in this work because it describes pressure dependent dynamics, ${ }^{6,7}$ which means it is capable of resolving a material's response to density change (which we connect to interfacial effects) independently from its response to temperature change. In accord with experimental observation, $\mathrm{CFV}$ predicts that the dynamics of a material will follow a general form,

$$
\tau(T, V) \propto \exp [f(T) \times g(V)] .
$$

where the temperature contribution, $f(T)$, and the volume (density) contribution, $g(V)$, are multiplicatively coupled.

In CFV, the volume contribution is described in terms of the free volume, $V_{\text {free, }}$ which is the most efficient variable to use because all systems will follow the same form, $g(V) \propto 1 / V_{\text {free. }}$. We have shown this correlation for many systems $\mathrm{s}^{1,3,5,8}$ and emphasize that it does not involve any fitting of $V_{\text {free }}$ to the dynamics data. The calculation of $V_{\text {free }}$ is based on an independent a priori analysis of the material's pressure, volume, temperature $(P V T)$ data using the locally correlated lattice (LCL) equation of state (EOS), ${ }^{9}$ where for any bulk material we define

$$
V_{\text {free }}=V-V_{\mathrm{hc}}
$$

$V$ is the system's total volume at any given $T, P$. The constant, $V_{\mathrm{hc}}$, is its volume in the limiting closely packed state, and is calculated from the LCL EOS molecular parameters, which relate to the size of the molecules. (For the interested reader, ref $^{5}$ describes another very simple route to the close-packed volume from $P V T$ data.) From the LCL EOS fit to the P2ClS PVT data, ${ }^{10}$ we find $V_{\mathrm{hc}}=0.733 \mathrm{~mL} / \mathrm{g}$. The LCL application to P2ClS, including the expression for $P(V, T)$ and its molecular level parameters, has been covered in detail in the supporting information for ref ${ }^{11}$.

In accordance with eq $\mathrm{S} 1$, the $\mathrm{CFV}$ working expression for relaxation times is given by

$$
\ln \tau=\left(\frac{V_{\mathrm{hc}}}{V_{\text {free }}}\right)\left(\frac{T^{*}}{T}\right)^{b}+\ln \tau_{\text {ref }}
$$

The material specific parameters, $b, T^{*}, \tau_{\text {ref, }}$ are determined from bulk system dynamics data; alternatively $b$ can also be determined using $T_{\mathrm{g}}(P)$ from $P V T$ data. Using $\tau(T, P)$ data on bulk P2ClS from Schwartz, et al., ${ }^{12}$ and the corresponding $V_{\text {free }}(T, P)$ values from the above LCL EOS analysis, a fit of eq S3 leads to the parameters: $b=3.30, T^{*}=568.1 \mathrm{~K}, \log \tau_{\text {ref }}(/ \mathrm{s})=-11.36$. For those interested, ref ${ }^{11}$ provides a detailed discussion and analysis of the bulk P2ClS pressure dependent dynamics. Therein, model curves and data are shown in the form of Arrhenius plots $(\log \tau$ vs. $1 / T)$ at multiple pressures, and also demonstrated, is the linear correlation that results when the same data is plotted as $\log \tau$ vs. $1 / V_{\text {free }}$ on isotherms wherein each line (isotherm) has a slope that increases with decreasing $T$, which is the qualitative form of behavior predicted by CFV.

Equation S3 is also applied to model the capped and freestanding P2ClS films, and we do this using the very same values for the parameters, $b, T^{*}, \tau_{\text {ref, }}$ that apply to the P2ClS bulk. As we 
have discussed previously, ${ }^{2,4,13,14}$ this transferability means that the same temperature contribution, $f(T)\left(\propto\left(T^{*} / T\right)^{b}\right)$, that works for the bulk material also works, at least approximately, for the film. Though the $f(T)$ contribution is the same, one should expect that the sample-averaged relative free volume for a film (denoted $\left(V_{\text {free }} / V_{\text {hc }}\right)_{\text {film }}$ ) will be different from that of the corresponding bulk at the same $T, P$ (denoted $V_{\text {free }} / V_{\text {hc }}$ ) bulk) due to the additional free space near a film's interface. To account for this we apply a simple two layer picture to the overall sample (details of the derivation are in $\mathrm{ref}^{2}$ ). Here we consider the average relative free volume in an (inhomogeneous) interfacial region and that in an interior bulk-like region, and take their weighted average; the thinner the film, the larger the relative weight of the interfacial region. The resulting overall sample average, $\left(V_{\text {free }} / V_{\mathrm{hc}}\right)_{\text {film, }}$, is given by

$$
\left(V_{\text {free }} / V_{\text {hc }}\right)_{\text {film }}=\left(V_{\text {free }} / V_{\text {hc }}\right)_{\text {bulk }}+\left(\delta_{\text {free }} / h\right)
$$

where the temperature- and thickness- independent parameter, $\delta_{\text {free}}$, characterizes the excess of free volume in the interfacial region relative to that in the bulk. As discussed in the text of the main article, $\delta_{\text {free }}$ will be different depending on whether it corresponds to a freestanding film or a capped film. (In the latter case it will also depend on, and thus characterize, the particular degree to which the polymer is adsorbed). The only requirement of eq S4 is that it is not applied to a film so thin that a bulk-like region doesn't exist; we found in ref $^{13}$ that this breakdown point for the model occurs for films that are thinner than $10 \mathrm{~nm}$, and so we stay above this limit in the present work.

To summarize, the modeling proceedure for films involves calculating the eq S4 result for the film at the chosen $T$ (and $P=1 \mathrm{~atm}$ ) based on the (fitted) value of $\delta_{\text {free, }}$ the value of the film thickness, $h$, and the value of $\left(V_{\text {free }} / V_{\mathrm{hc}}\right)$ bulk for that given $T$ (and $P=1 \mathrm{~atm}$ ). Then, this resulting ( $V_{\text {free }} / V_{\text {hc }}$ ) film value (along with the given $T$ ) is input into eq $\mathrm{S} 3$ to calculate the film relaxation time, $\tau(T, h)$. As discussed in the main text, the value of $\delta_{\text {free }}$ (at least for capped films) cannot be obtained a priori from bulk information alone, so in practice, the order of the calculations is to first use experimentally measured $\tau$ values and corresponding $T$ values for the films in eq S3 to effectively solve for $\left(V_{\text {free }} / V_{\text {hc }}\right)_{\text {film }}$, and this leads to the best/fitted value for $\delta_{\text {free }}$ according to eq $\mathrm{S} 4$. It is important to note that obtaining a fitted value for $\delta_{\text {free }}$ could require as little as a single film datum point, corresponding to a single film of thickness, $h$, measured at a single temperature (in cases where the experimental uncertainty is low), and so in principle, it is then possible to predict the dynamics $(\tau)$ at all other $T$ and $h$ values because the simple $\delta_{\text {free }}$ parameter is independent of both temperature and thickness.

Finally, the following are some details on the predictions discussed in the main article regarding how much pressure one would need to apply to a film in order to decrease its free volume to the point where it matches the material's bulk dynamics. In the $T$ and $P$ regime of the data shown in the figures of the main article, the pressure dependence on relative free volume, $V_{\text {free }} / V_{\text {hc }}$, is approximately $\mathrm{d} P / \mathrm{d}\left(V_{\text {free }} / V_{\mathrm{hc}}\right)=-1333 \mathrm{MPa}$. Given the $\delta_{\text {free }}$ values, 0.20 and $0.46 \mathrm{~nm}$, found for capped and freestanding films respectively, we have the following: the difference in relative free volume between the $18 \mathrm{~nm}$ capped film and the bulk at ambient pressure is $\delta_{\text {free }} / h=0.0111$, and that between the $18 \mathrm{~nm}$ freestanding film and bulk is $\delta_{\text {free }} / h=0.0256$, and the difference between the 18 freestanding and 18 capped films is $\Delta \delta_{\text {free }} / h=0.0144$. Based on this, the $18 \mathrm{~nm}$ capped film would require a sample pressure increase of $14.8 \mathrm{MPa}$ for its free volume to be reduced to that of the ambient pressure bulk (at the same $T$ ) and thus obtain bulk dynamics. The $18 \mathrm{~nm}$ 
freestanding film, which has more free volume than the $18 \mathrm{~nm}$ capped film, would require a pressure increase of $19.3 \mathrm{MPa}$ for it to obtain the same dynamics as the $18 \mathrm{~nm}$ capped film, and a further increase of 14.8 , so $34.1 \mathrm{MPa}$ in total, in order for it to reach the dynamics of the ambient bulk.

\section{References}

(1) White, R. P.; Lipson, J. E. G. Explaining the T,V-Dependent Dynamics of Glass Forming Liquids: The Cooperative Free Volume Model Tested against New Simulation Results. J. Chem. Phys. 2017, 147 (18), 184503-184503. https://doi.org/10.1063/1.5001714.

(2) White, R. P.; Lipson, J. E. G. Connecting Pressure-Dependent Dynamics to Dynamics under Confinement: The Cooperative Free Volume Model Applied to Poly(4Chlorostyrene) Bulk and Thin Films. Macromolecules 2018, 51 (20), 7924-7941. https://doi.org/10.1021/acs.macromol.8b01392.

(3) White, R. P.; Lipson, J. E. G. The Cooperative Free Volume Rate Model for Segmental Dynamics: Application to Glass-Forming Liquids and Connections with the Density Scaling Approach. Eur. Phys. J. E 2019, 42 (8), 100. https://doi.org/10.1140/epje/i201911862-3.

(4) White, R. P.; Lipson, J. E. G. To Understand Film Dynamics Look to the Bulk. Phys. Rev. Lett. 2020, 125 (5), 058002. https://doi.org/10.1103/PhysRevLett.125.058002.

(5) White, R. P.; Lipson, J. E. G. A Simple New Way To Account for Free Volume in Glassy Dynamics: Model-Free Estimation of the Close-Packed Volume from PVT Data. J. Phys. Chem. B 2021, 125 (16), 4221-4231. https://doi.org/10.1021/acs.jpcb.1c01620.

(6) Roland, C.; Hensel-Bielowka, S.; Paluch, M.; Casalini, R. Supercooled Dynamics of Glass-Forming Liquids and Polymers under Hydrostatic Pressure. Rep. Prog. Phys. 2005, 68 (6), 1405-1478. https://doi.org/10.1088/0034-4885/68/6/R03.

(7) Floudas, G.; Paluch, M.; Grzybowski, A.; Ngai, K. Molecular Dynamics of Glass-Forming Systems - Effects of Pressure; Springer: Berlin, 2011.

(8) White, R. P.; Lipson, J. E. G. How Free Volume Does Influence the Dynamics of Glass Forming Liquids. Acs Macro Lett. 2017, 6 (5), 529-534.

https://doi.org/10.1021/acsmacrolett.7b00179.

(9) White, R. P.; Lipson, J. E. G. Polymer Free Volume and Its Connection to the Glass Transition. Macromolecules 2016, 49 (11), 3987-4007. https://doi.org/10.1021/acs.macromol.6b00215.

(10) Roland, C. M.; McGrath, K. J.; Casalini, R. Dynamic Heterogeneity in Poly(Vinyl Methyl Ether)/Poly(2-Chlorostyrene) Blends. Macromolecules 2006, 39 (10), 3581-3587. https://doi.org/10.1021/ma060315k.

(11) White, R. P.; Lipson, J. E. G. The Dynamics of Freestanding Films: Predictions for Poly(2-Chlorostyrene) Based on Bulk Pressure Dependence and Thoughtful Sample Averaging. Soft Matter 2021. https://doi.org/10.1039/D1SM01175H.

(12) Schwartz, G. A.; Colmenero, J.; Alegria, A. Pressure-Temperature Dependence of Polymer Segmental Dynamics. Comparison between the Adam-Gibbs Approach and Density Scalings. Macromolecules 2006, 39 (11), 3931-3938. https://doi.org/10.1021/ma052464t.

(13) Debot, A.; White, R. P.; Lipson, J. E. G.; Napolitano, S. Experimental Test of the Cooperative Free Volume Rate Model under 1D Confinement: The Interplay of Free 
Volume, Temperature, and Polymer Film Thickness in Driving Segmental Mobility. Acs Macro Lett. 2019, 8 (1), 41-45. https://doi.org/10.1021/acsmacrolett.8b00844.

(14) White, R. P.; Lipson, J. E. G. Dynamics across a Free Surface Reflect Interplay between Density and Cooperative Length: Application to Polystyrene. Macromolecules 2021, 54 (9), 4136-4144. https://doi.org/10.1021/acs.macromol.0c02742. 